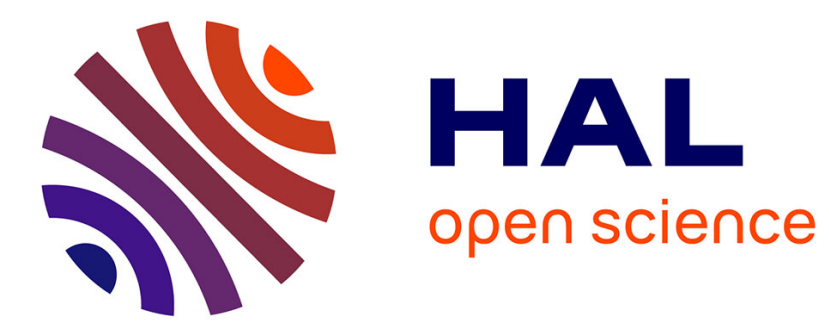

\title{
Specular and diffuse reflections of rays in coupled thin plates at high frequencies
}

\author{
Alain Le Bot, Vincent Cotoni
}

\section{To cite this version:}

Alain Le Bot, Vincent Cotoni. Specular and diffuse reflections of rays in coupled thin plates at high frequencies. Journal of Sound and Vibration, 2003, 265 (1), pp.23-41. hal-00469441

\section{HAL Id: hal-00469441 \\ https://hal.science/hal-00469441}

Submitted on 1 Apr 2010

HAL is a multi-disciplinary open access archive for the deposit and dissemination of scientific research documents, whether they are published or not. The documents may come from teaching and research institutions in France or abroad, or from public or private research centers.
L'archive ouverte pluridisciplinaire HAL, est destinée au dépôt et à la diffusion de documents scientifiques de niveau recherche, publiés ou non, émanant des établissements d'enseignement et de recherche français ou étrangers, des laboratoires publics ou privés. 


\title{
Specular and diffuse reflections of rays in coupled thin plates at high freqeuncies
}

\author{
V. COTONI \\ Groupe d'Acoustique de l'Université de Sherbrooke \\ Université de Sherbrooke, CANADA \\ A. LE BOT \\ Laboratoire de Tribologie et Dynamique des Systèmes CNRS \\ École centrale de Lyon, FRANCE alain.le-bot@ec-lyon.fr
}

\begin{abstract}
The flexural energy distribution in two right-angled point-excited thin plates at high frequencies is investigated by means of an integral energy flow approach. Time and frequencyaveraged energy fields are described by the superposition of uncorrelated cylindrical waves stemming from both boundaries and direct sources. Specular and diffuse laws are considered for the reflection and transmission of rays, giving rise to two kinds of energy equations. The diffuse law leads to a Fredholm integral equation while the specular law is shown to allow an image source solution when the plates have identical propagation properties. The algorithm for computing the image position, magnitude and directivities is described. Then, some comparisons between the results from the two energy formulations and also from the Statistical Energy Analysis and the numerical solving of the equations of motion are performed with a couple of damped plates at high frequency. The non diffuse pattern of the averaged flexural energy fields is well described by the energy flow approaches.
\end{abstract}

\section{Introduction}

One is here interested in the prediction of vibratory levels of systems of damped thin plates in the high frequency range. Due to system uncertainties and the high computation cost, classical finite element methods are not suitable for high frequencies. By mean of statistical hypothesis, energy approaches provide an efficient tool for the evaluation of averaged energy levels. However, the most commonly used, the Statistical Energy Analysis (SEA) [1] assumes that energy fields are diffuse and does not give the energy distribution inside systems, but only its averaged value. It consequently leads to poor results when systems are highly damped and, therefore, when energy fields are non-diffuse.

Several investigations have been performed to contravene this feature. The conductivity approach initiated by Nefske et al. [2] and developed by Bouthier et al. [3] is based on a plane waves decomposition and the diffuse directivity of energy is assumed for both incidence and emission on boundaries. It is shown to be reliable for lightly damped systems only where energy fields are slightly non-diffuse [4, 5]. Kuttruff [6], Miles [7] and Le Bot [8] presented an integral energy 


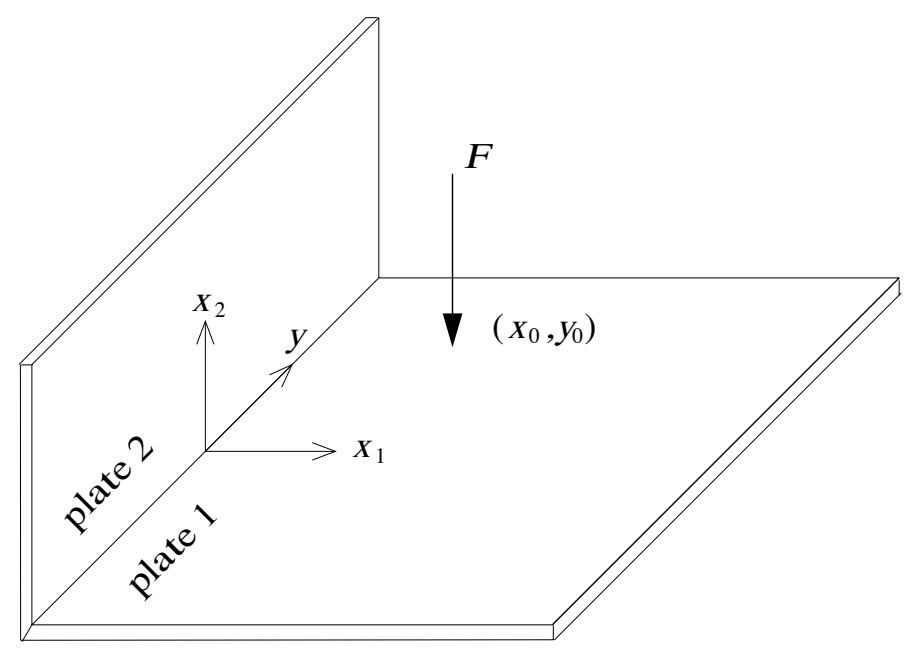

Figure 1: Two right-angled plates. The plate 1 is excited by a point force.

approach using spherical waves in acoustics and cylindrical waves for plates, and assuming the diffuse law for the reflection of rays. It proves to accurately predict the non diffuse energy fields of acoustical enclosures [9] and highly damped plates [10,11]. A similar approach using specular laws for the emission was also developed by Franzoni et al. [12] and Le Bot [13]. Cotoni et al. applied this approach to radiation problems and obtained an accurate description of the non diffuse exterior acoustic field [10, 14]. Recently, Chae et al. [15] applied a ray tracing method using specular reflection law and ray tube concepts. They focused on the ability of the approach to account for the filtering effect of junctions, that is all incidences are not similarly transmitted and reflected.

Very little work has been published on the differences and similarities of energy approaches using diffuse and specular reflections of energetic rays [16]. This paper is concerned with such a comparison on a system of right-angled plates with particular stress on the influence of specular and diffuse directivities to describe the filtering effect of the junction. The propagation of the flexural energy with cylindrical rays is first addressed. Then, both specular and diffuse directivities are investigated to characterize the boundary energy conditions of each plate. It is shown how the image source method may be applied to solve the specular energy equation for coupled plates. Finally, some comparisons are performed with SEA results and reference results from the solving of the equations of motion.

\section{Description of flexural energy fields}

The integral energy flow approach developed in references $[8,11,13]$ is applied to the system of two right-angled plates shown in Figure 1. A complete description of dynamics in plates in terms of rays as presented in reference [11] requires taking into account both out-of-plane and in-plane motions. But, for a transversely excited thin plate, the out-of-plane motion dominates the dynamics and, therefore, for the sake of simplicity we shall consider that in-plane motion is negligible. This section describes the flexural energy field in plates with a decomposition in cylindrical traveling waves. The energy field for a single cylindrical wave is first derived. Then, the complete energy 
field is obtained by super-imposing these elementary fields. Two boundary conditions are derived depending on the kind of reflection-transmission law adopted for rays at the interface between plates.

\subsection{Energy propagation}

Consider a homogeneous plate, damped with the loss factor $\eta$, under harmonic excitation at pulsation $\omega$. We seek to determine the time-averaged flexural energy of the direct field at any point $\mathbf{r}$, due to a point source of unit power being injected on point $\mathbf{s}$. Since evanescent waves do not carry any power and rapidly vanish at high frequencies, the approximate direct energy density $G$ and intensity vector $\mathbf{H}$ are estimated by considering only the traveling wave contribution, $[8,13]$

$$
G(\mathbf{s}, \mathbf{r})=\frac{1}{c} \frac{e^{-m r}}{2 \pi r}, \quad \mathbf{H}(\mathbf{s}, \mathbf{r})=\frac{e^{-m r}}{2 \pi r} \mathbf{u}_{\mathbf{s r}},
$$

where $m=\eta \omega / c$ is the attenuation coefficient and $c$ is the group speed of flexural waves. $r=|\mathbf{r}-\mathbf{s}|$ is the distance between $\mathbf{s}$ and $\mathbf{r}$ and $\mathbf{u}_{\mathbf{s r}}=(\mathbf{r}-\mathbf{s}) / r$ is the unit vector in the direction from $\mathbf{s}$ to $\mathbf{r}$.

The direct field is reflected when impinging on the boundary $\Gamma$ of the plate, giving rise to new traveling waves. Thus, assuming that all traveling waves are uncorrelated, the complete energy density $W$ and intensity vector $\mathbf{I}$ are the sum of the contributions of the direct sources $\mathbf{s}$ with magnitude $p_{\text {inj }}$ and the boundary sources $\mathbf{p}$ with magnitude $\sigma$,

$$
\begin{aligned}
& W(\mathbf{r})=\int_{\Omega} p_{\text {inj }}(\mathbf{s}) G(\mathbf{s}, \mathbf{r}) d \Omega_{\mathbf{s}}+\int_{\Gamma} \sigma\left(\mathbf{p}, \theta_{\mathbf{p}}\right) G(\mathbf{p}, \mathbf{r}) d \Gamma_{\mathbf{p}}, \\
& \mathbf{I}(\mathbf{r})=\int_{\Omega} p_{\text {inj }}(\mathbf{s}) \mathbf{H}(\mathbf{s}, \mathbf{r}) d \Omega_{\mathbf{s}}+\int_{\Gamma} \sigma\left(\mathbf{p}, \theta_{\mathbf{p}}\right) \mathbf{H}(\mathbf{p}, \mathbf{r}) d \Gamma_{\mathbf{p}} .
\end{aligned}
$$

where $\Omega$ is the domain of the plate, $\Gamma$ the boundary and $\theta_{\mathbf{p}}$ the emission angle at $\mathbf{p}$ towards $\mathbf{r}$. The first integral in each expression is the direct field, the second is the reflected field. Notice that the boundary sources $\sigma$ may have a non-uniform directivity. The magnitude $p_{\text {inj }}$ of direct sources is the time-averaged density of power being injected. For the case of a single driving force at point $\mathbf{s}_{0}$, it is written $p_{\text {inj }}(\mathbf{s})=P_{\text {inj }} \delta_{\mathbf{s}_{0}}$ with $P_{\text {inj }}$ being the time-averaged injected power attached to the direct field. It is derived from the infinite thin plate solution [17], for an applied force $F$ with a bending stiffness $D$, and a mass per unit surface $m$,

$$
P_{\text {inj }}=F^{2} / 16 \sqrt{D m} .
$$

Now, the density of source $\sigma$ is to be evaluated, by expressing the appropriate energy boundary conditions. At this stage, two assumptions may be made on the way energetic rays are reflected and transmitted. Specular or diffuse laws lead to different forms of energy integral equations. In the following, all energy quantities are now written with a subscript $i, j=1,2$ of the corresponding plate.

\subsection{Energy reflection and transmission}

Any boundary is characterized in terms of energy balance by efficiencies that are the ratios of reflected and transmitted powers over the incident one. They are usually derived according to 


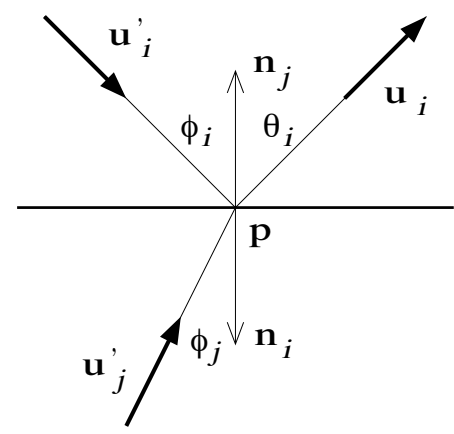

Figure 2: Power balance at the point $\mathbf{p}$ of the interface between plates $i$ and $j$.

the locality principle valid at high frequencies [18] which states that the dynamics of the coupling only depends on the local properties of the system in the vicinity of the coupling. The underlying assumption is that the wavelength is small enough so that the boundary appears locally straight and homogeneous. It follows that diffraction effects are not considered in the present analysis. For the case of two coupled plates of Figure 1, the junction is characterized by a reflection efficiency $R_{i}(\phi)$ and a transmission efficiency $T_{i}(\phi)$ depending on the incidence angle $\phi$. The subscript $i$ denotes the plate of incidence. Explicit values for $R_{i}$ and $T_{i}$ may be found in reference [19]. If the other boundaries of the plates are perfectly reflective boundaries (like simply supported, free or clamped edges) they are characterized by the uniform reflection efficiency $R_{i}(\phi)=1$.

The most general case of junction is now presented. Following notations of Figure 2, let $\mathbf{u}_{i}$ denote the emission direction with emission angle $\theta_{i}, \mathbf{u}_{i}^{\prime}$ and $\mathbf{u}_{j}^{\prime}$ the incident directions from both plates with incidences $\phi_{i}, \phi_{j}$, and $\mathbf{n}_{i}$ the outside unit normal to the boundary of plate $i$.

The power balance at any point $\mathbf{p}$ of the junction is written

$$
\mathcal{P}_{i, \text { emit }}\left(\mathbf{u}_{i}\right) d \theta_{i}=R_{i}\left(\phi_{i}\right) \mathcal{P}_{i, \text { inc }}\left(\mathbf{u}_{i}^{\prime}\right) d \phi_{i}+T_{j}\left(\phi_{j}\right) \mathcal{P}_{j, \text { inc }}\left(\mathbf{u}_{j}^{\prime}\right) d \phi_{j},
$$

where $\mathcal{P}_{i \text {,inc }}\left(\mathbf{u}_{i}^{\prime}\right)$ denotes the incident power at $\mathbf{p}$ stemming from the direction $\mathbf{u}_{i}^{\prime}$ and $\mathcal{P}_{i, \text { emit }}\left(\mathbf{u}_{i}\right)$ the emitted power in direction $\mathbf{u}_{i}$. $d \theta_{i}$ is an infinitesimal angle of emitted directions about $\mathbf{u}_{i}, d \phi_{i}$ the corresponding angles of incident directions from plate $i$ and $d \phi_{j}$ the corresponding angles of incident directions from plate $j$. These powers may be expressed in terms of the direct sources $p_{i, \text { inj }}$ and the diffracted sources $\sigma_{i}$ of equation (3).

Considering first the diffuse law for reflection and transmission [20], any ray impinging on the boundary is assumed to be reflected and transmitted with the Lambert directivity i.e. with a magnitude proportional to the cosine of the emission angle, whatever is the incident angle. The magnitude of the boundary sources $\sigma_{i}(\mathbf{p}, \theta)$ may be factorized in $\sigma_{i}(\mathbf{p}) \cos \theta$. By integrating equation (5) over all emission angles and related incident angles, a Fredholm equation of second kind on the unknowns $\sigma_{i}$ is obtained $[8,9,11]$,

$$
\begin{aligned}
\frac{\sigma_{i}(\mathbf{p})}{\pi}=\left[\int_{\Omega_{i}} R_{i}\left(\phi_{\mathbf{s}}\right) p_{i, \mathrm{inj}}(\mathbf{s}) \mathbf{H}_{i}(\mathbf{s}, \mathbf{p}) d \Omega_{\mathbf{s}}+\int_{\Gamma_{i}} R_{i}\left(\phi_{\mathbf{q}}\right) \sigma_{i}(\mathbf{q}) \cos \theta_{\mathbf{q}} \mathbf{H}_{i}(\mathbf{q}, \mathbf{p}) d \Gamma_{\mathbf{q}}\right] . \mathbf{n}_{i}+ \\
{\left[\int_{\Omega_{j}} T_{j}\left(\phi_{\mathbf{s}}\right) p_{j, \text { inj }}(\mathbf{s}) \mathbf{H}_{j}(\mathbf{s}, \mathbf{p}) d \Omega_{\mathbf{s}}+\int_{\Gamma_{j}} T_{j}\left(\phi_{\mathbf{q}}\right) \sigma_{j}(\mathbf{q}) \cos \theta_{\mathbf{q}} \mathbf{H}_{j}(\mathbf{q}, \mathbf{p}) d \Gamma_{\mathbf{q}}\right] \cdot \mathbf{n}_{j} . }
\end{aligned}
$$


where $\theta_{\mathbf{q}}$ is the emission angle at $\mathbf{q}$ towards $\mathbf{p}$ and $\phi_{\mathbf{s}}$ (resp. $\phi_{\mathbf{q}}$ ) the incident angle at $\mathbf{p}$ from $\mathbf{s}$ (resp. q). The terms in the first brackets are the reflected power from plate $i$, the second brackets the transmitted power from plate $j$ to plate $i$. Note that although the emitting directivity is postulated following the Lambert law, incidence is not assumed to be diffuse and the directional efficiencies $R_{i}(\phi)$ and $T_{j}(\phi)$ are used in equation (6) and not the mean-efficiency $\bar{T}=\int_{0}^{\pi / 2} T(\phi) \cos (\phi) d \phi$ usually preferred in SEA. Equation (6) may be solved with a collocation algorithm as detailed in section 3. Substituting its solutions for $\sigma_{1}$ and $\sigma_{2}$ in equation (2) gives the energy field in each plate.

Consider now that the specular law for reflection and transmission applies for rays. The power balance (5) is applied direction by direction. The emission angle $\theta_{i}$ is now related to the incident angle $\phi_{j}$ by the Snell law of refraction $\sin \theta_{i} / c_{i}^{\prime}=\sin \phi_{j} / c_{j}^{\prime}$ where $c_{i}^{\prime}$ is the phase speed in plate $i$. Indeed $\phi_{i}=\theta_{i}$ and we also introduce $\theta_{j}=\phi_{j}$. Incident angles $\phi_{i}, \phi_{j}$ are no longer necessary and notations $\theta_{i}, \theta_{j}$ are now preferred. The points $\mathbf{p}_{i}^{\prime}$ are defined as being the point lying in the boundary $\Gamma_{i}$ in such a manner that the point $\mathbf{p}$ is viewed from $\mathbf{p}_{i}^{\prime}$ in direction $\mathbf{u}_{i}^{\prime}$. The emission angle at $\mathbf{p}_{i}^{\prime}$ is noted $\theta_{i}^{\prime}$. From reference [13], the functional equation for the diffraction sources $\sigma_{i}$ located on each side of the interface at any point $\mathbf{p}$ is

$$
\begin{aligned}
\frac{\sigma_{i}\left(\mathbf{p}, \theta_{i}\right)}{\cos \theta_{i}}=R_{i}\left(\theta_{i}\right)\left[\frac{\sigma_{i}\left(\mathbf{p}_{i}^{\prime}, \theta_{i}^{\prime}\right)}{\cos \theta_{i}^{\prime}} e^{-m_{i} r_{i}^{\prime}}\right. & \left.+\int_{\mathbf{p}_{i}^{\prime} \mathbf{p}} p_{i, \mathrm{inj}}(\mathbf{s}) e^{-m_{i} s} d s\right] \\
& +\frac{c_{j}^{\prime}}{c_{i}^{\prime}} T_{j}\left(\theta_{j}\right)\left[\frac{\sigma_{j}\left(\mathbf{p}_{j}^{\prime}, \theta_{j}^{\prime}\right)}{\cos \theta_{j}^{\prime}} e^{-m_{j} r_{j}^{\prime}}+\int_{\mathbf{p}_{j}^{\prime} \mathbf{p}} p_{j, \mathrm{inj}}(\mathbf{s}) e^{-m_{j} s} d s\right],
\end{aligned}
$$

where $r_{i}^{\prime}=\left|\mathbf{p}_{i}^{\prime}-\mathbf{p}\right|, s=|\mathbf{s}-\mathbf{p}|$. The first terms in each bracket is the contribution of other boundary sources $\sigma_{i}$ modified with the attenuation term $e^{-m_{i} r_{i}^{\prime}}$, whereas the second term, an integral over the path $\mathbf{p}_{i}^{\prime} \mathbf{p}$ is the contribution of actual sources $p_{i, \text { inj }}$ located inside the plate $i$. The points $\mathbf{p}_{i}^{\prime}$ may not exist in some particular situations like semi-infinite plates. In such a case the corresponding terms in the previous equation must be canceled and the integral for actual sources is extended to a semi-infinite line $\infty \mathbf{p}$. The functional equation (7) may be solved by the image source method when the plates have identical propagation properties, as will be shown in section 4.

\subsection{Application limits}

Integral energy approaches are based on the three assumptions that $i$ ) vibrating fields are composed of rays, ii) these rays are uncorrelated, iii) couplings are local phenomena. The first and third assumptions are those invoked by classical ray methods so that a similar validity range is expected for the integral energy approaches: the wavelength must be small compared to the characteristic dimensions of the systems. This condition is well suited as frequency increases meaning that the approach is confined to middle and high frequencies. Another consequence is that near fields are neglected leading to an incorrect description of energy in the neighborhood of discontinuities. The second assumption is related to the uncertainty on the phase of rays at high frequency, which leads to the use of energy variables as in SEA [1]. The validity in terms of frequency will consequently be similar as that of SEA. However, no assumption has been made on the diffuse nature of the vibrating fields so that the integral energy approaches allow to extend the SEA prediction to systems with non diffuse fields. 


\section{Collocation solution for the integral equation (6)}

The integral equation (6) is solved for the couple of square plates of Figure 1 by using the collocation method. A special software named CeReS has been developed to achieve this task for various problems involving assembled plates of arbitrary shape.

The method for the resolution is as follows. Each edge of plates is divided into a limited number of elements $L_{k}, k=1,2 \ldots$ of equal size. The magnitude $\sigma_{i}$ of the boundary sources is assumed to be constant over each boundary element. The unknown magnitude attached to the boundary element numbered $k$ is noted $\sigma_{i}^{k}$. For each element $k$, equation (6), depending on the position of the element at edge or interface, is applied at the middle $\mathbf{p}_{k}$ of the element. This point $\mathbf{p}_{k}$ is called the collocation point. First, for an element $k$ located at the edge of plate $i$,

$$
\sigma_{i}^{k}=\pi\left[P_{\mathrm{i}, \text { inj }} H_{i}\left(\mathbf{s}_{0}, \mathbf{p}_{k}\right) \cos \phi_{\mathbf{s}_{0}}+\sum_{l} \sigma_{i}^{l} \int_{L_{l}} H_{i}\left(\mathbf{q}, \mathbf{p}_{k}\right) \cos \theta_{\mathbf{q}} \cos \phi_{\mathbf{q}} d \Gamma_{\mathbf{q}}\right],
$$

where $\phi_{\mathbf{s}_{0}}$ is the incidence angle at the collocation point $\mathbf{p}_{k}$ from the actual source $\mathbf{s}_{0}, \phi_{\mathbf{q}}$ is the incidence angle at $\mathbf{p}_{k}$ from $\mathbf{q}$ and $\theta_{\mathbf{q}}$ the emanating direction at $\mathbf{q} . H_{i}$ is the magnitude of the intensity $\mathbf{H}_{i}$. The first term inside the brackets is the direct contribution of the actual source, and is to be considered for plate 1 only since plate 2 is not directly excited $\left(P_{2, \text { inj }}=0\right)$. The second sum runs over all boundary elements of the plate. Second, for an element located at the interface between the two plates,

$$
\begin{array}{r}
\sigma_{i}^{k}=\pi\left[P_{\mathrm{i}, \mathrm{inj}} R_{i}\left(\phi_{\mathbf{s}_{0}}\right) H_{i}\left(\mathbf{s}_{0}, \mathbf{p}_{k}\right) \cos \phi_{\mathbf{s}_{0}}+\sum_{l} \sigma_{i}^{l} \int_{L_{l}} R_{i}\left(\phi_{\mathbf{q}}\right) H_{i}\left(\mathbf{q}, \mathbf{p}_{k}\right) \cos \theta_{\mathbf{q}} \cos \phi_{\mathbf{q}} d \Gamma_{\mathbf{q}}+\right. \\
\left.P_{\mathrm{j}, \text { inj }} T_{j}\left(\phi_{\mathbf{s}_{0}}\right) H_{j}\left(\mathbf{s}_{0}, \mathbf{p}_{k}\right) \cos \phi_{\mathbf{s}_{0}}+\sum_{l} \sigma_{j}^{l} \int_{L_{l}} T_{j}\left(\phi_{\mathbf{q}}\right) H_{j}\left(\mathbf{q}, \mathbf{p}_{k}\right) \cos \theta_{\mathbf{q}} \cos \phi_{\mathbf{q}} d \Gamma_{\mathbf{q}}\right] .
\end{array}
$$

Thus, the set of equations $(8,9)$ leads to a system of linear equations for the unknowns $\sigma_{i}^{k}$. The coefficients involve some integrals evaluated by Gauss quadrature. Once the source magnitudes $\sigma_{i}^{k}$ are computed, the energy density inside plate $i$ is evaluated from the discrete version of equation $(2)$,

$$
W_{i}(\mathbf{r})=P_{i, \text { inj }} G_{i}\left(\mathbf{s}_{0}, \mathbf{r}\right)+\sum_{k} \sigma_{i}^{k} \int_{L_{k}} G_{i}(\mathbf{p}, \mathbf{r}) \cos \theta_{\mathbf{p}} d \Gamma_{\mathbf{p}}
$$

In this expression, the sums of the right-hand side run over all sources acting on the plate where $\mathbf{r}$ lies.

\section{Image source solution for the functional equation (7)}

The problem is now to solve the functional equation (7) for the couple of square plates of Figure 1. Several methods are available, like the boundary element method proposed in [12], where the directivity of each source is discretized with a Fourier series expansion, or the ray tracing approach based on ray tubes developed in [15]. However, it will be shown that the current problem can be solved with the image source technique due to the simple geometry. We proceed in two steps. The first step is to solve it for the case of two semi-infinite plates separated by an infinite interface. A 


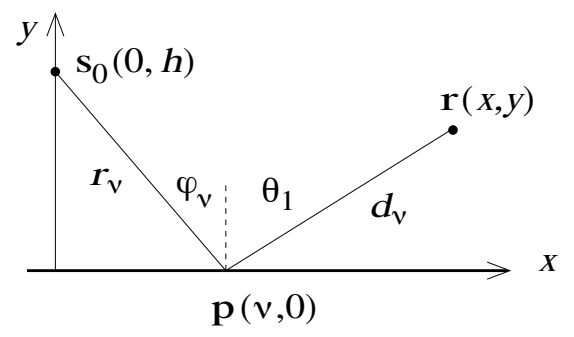

Figure 3: Notations for the specular law of transmission and reflection between two semi-infinite plates.

single excitation point acts in plate 1 . The second step is to apply the image source technique for the couple of square plates.

\subsection{Two semi-infinite plates}

First, consider a steady-state source point $\mathbf{s}_{0}(0, h)$ within plate 1 and a point $\mathbf{p}(\nu, 0)$ lying on the interface whose equation is $y=0$ (see Figure 3 ). The actual source density is written $p_{\text {inj }}(\mathbf{s})=$ $P_{\mathrm{inj}} \delta_{\mathrm{s}_{0}}$. where $P_{\mathrm{inj}}$ is given in equation (4). Both plates are considered semi-infinite, and the functional equation (7) for the interface reduces to

$$
\begin{aligned}
\frac{\sigma_{1}\left(\nu, \theta_{1}\right)}{\cos \theta_{1}} & =R_{1}\left(\theta_{1}\right) \int_{\infty \mathbf{p}} P_{\mathrm{inj}} \delta_{\mathbf{s}_{0}}(\mathbf{s}) e^{-m_{1} s} d s, \\
\frac{\sigma_{2}\left(\nu, \theta_{2}\right)}{\cos \theta_{2}} & =\frac{c_{1}^{\prime}}{c_{2}^{\prime}} T_{1}\left(\theta_{1}\right) \int_{\infty \mathbf{p}} P_{\mathrm{inj}} \delta_{\mathbf{s}_{0}}(\mathbf{s}) e^{-m_{1} s} d s,
\end{aligned}
$$

The delta Dirac function may be expanded in polar coordinates $(s, \theta)$ centered at $\mathbf{p}$,

$$
\delta_{\mathbf{s}_{0}}(\mathbf{s})=\frac{\delta_{r_{\nu}}(s) \delta_{\varphi_{\nu}}(\theta)}{s},
$$

where $r_{\nu}=\left|\mathbf{s}_{0}-\mathbf{p}\right|=\left[\nu^{2}+h^{2}\right]^{1 / 2}$ and $\varphi_{\nu}$ is the incident angle at $\mathbf{p}$ from $\mathbf{s}_{0}$. It leads to the solutions

$$
\begin{aligned}
& \frac{\sigma_{1}\left(\nu, \theta_{1}\right)}{\cos \theta_{1}}=P_{\mathrm{inj}} R_{1}\left(\theta_{1}\right) \frac{e^{-m_{1} r_{\nu}}}{r_{\nu}} \delta_{\varphi_{\nu}}\left(\theta_{1}\right), \\
& \frac{\sigma_{2}\left(\nu, \theta_{2}\right)}{\cos \theta_{2}}=P_{\mathrm{inj}} \frac{c_{1}^{\prime}}{c_{2}^{\prime}} T_{1}\left(\theta_{1}\right) \frac{e^{-m_{1} r_{\nu}}}{r_{\nu}} \delta_{\varphi_{\nu}}\left(\theta_{1}\right) .
\end{aligned}
$$

In the second equality, the angles $\theta_{1}$ and $\theta_{2}$ are related each other with the Snell law for refraction.

Let now evaluate the energy fields inside both plates. First, let $\mathbf{r}(x, y)$ be any point in plate 1 . Following equation (2) the energy density $W_{1}$ is found to be

$$
W_{1}(\mathbf{r})=P_{\mathrm{inj}} \frac{e^{-m_{1} q_{0}}}{2 \pi c_{1} q_{0}}+\int_{-\infty}^{\infty} \sigma_{1}\left(\nu, \theta_{1}\right) \frac{e^{-m_{1} d_{\nu}}}{2 \pi c_{1} d_{\nu}} d \nu .
$$


where $q_{0}=\left|\mathbf{s}_{0}-\mathbf{r}\right|=\left[x^{2}+(y-h)^{2}\right]^{1 / 2}$ and $d_{\nu}=|\mathbf{p}-\mathbf{r}|=\left[(x-\nu)^{2}+y^{2}\right]^{1 / 2}$. $\theta_{1}$ is the emission angle at $\mathbf{p}$ towards $\mathbf{r}$. Substituting equation (14) into equation (16) gives

$$
W_{1}(\mathbf{r})=\frac{P_{\text {inj }}}{2 \pi c_{1}}\left[\frac{e^{-m_{1} q_{0}}}{q_{0}}+\int_{-\infty}^{\infty} R_{1}\left(\theta_{1}\right) \frac{e^{-m_{1} r_{\nu}}}{r_{\nu}} \delta_{\varphi_{\nu}}\left(\theta_{1}\right) \frac{e^{-m_{1} d_{\nu}}}{d_{\nu}} \cos \theta_{1} d \nu\right] .
$$

Now let denote $\psi_{\nu}=\theta_{1}-\varphi_{\nu}$. There exists one point $\mathbf{p}_{0}$ at $\nu_{0}$ which fulfills the condition $\theta_{1}=\varphi_{\nu_{0}}$. The emission angle $\theta_{1}$ is equal to the incident angle $\varphi_{\nu_{0}}$. Then,

$$
W_{1}(\mathbf{r})=\frac{P_{\text {inj }}}{2 \pi c_{1}}\left[\frac{e^{-m_{1} q_{0}}}{q_{0}}+R_{1}\left(\theta_{1}\right) \frac{e^{-m_{1} r_{0}}}{r_{0}} \frac{e^{-m_{1} d_{0}}}{d_{0}} \frac{\cos \theta_{1}}{\left|\frac{d \psi_{\nu}}{d \nu}\right|_{\nu=\nu_{0}}}\right],
$$

where $r_{0}=\left[\nu_{0}^{2}+h^{2}\right]^{1 / 2}$ and $d_{0}=\left[\left(x-\nu_{0}\right)^{2}+y^{2}\right]^{1 / 2}$ have been substituted for $r_{\nu_{0}}$ and $d_{\nu_{0}}$. Furthermore,

$$
\psi_{\nu}=\arctan \frac{\nu-x}{y}+\arctan \frac{\nu}{h} .
$$

By differentiating with respect to $\nu$,

$$
\frac{d \psi_{\nu}}{d \nu}=\frac{1 / y}{1+\left(\frac{\nu-x}{y}\right)^{2}}+\frac{1 / h}{1+\left(\frac{\nu}{h}\right)^{2}}=\frac{y}{d_{\nu}^{2}}+\frac{h}{r_{\nu}^{2}} .
$$

Since $y \geqslant 0$ and $h>0$, the derivative is never zero, and expression (18) is always defined. Then we obtain for $W_{1}$,

$$
W_{1}(\mathbf{r})=\frac{P_{\mathrm{inj}}}{2 \pi c_{1}}\left[\frac{e^{-m_{1} q_{0}}}{q_{0}}+R_{1}\left(\theta_{1}\right) \frac{e^{-m_{1} r_{0}}}{r_{0}} \frac{e^{-m_{1} d_{0}}}{d_{0}} \frac{\cos \theta_{1}}{\frac{y}{d_{0}^{2}}+\frac{h}{r_{0}^{2}}}\right] .
$$

Remarking that $\cos \theta_{1}=y / d_{0}=h / r_{0}$, it yields

$$
W_{1}(\mathbf{r})=\frac{P_{\mathrm{inj}}}{2 \pi c_{1}}\left[\frac{e^{-m_{1} q_{0}}}{q_{0}}+R_{1}\left(\theta_{1}\right) \frac{e^{-m_{1} q_{1}}}{q_{1}}\right],
$$

with $q_{1}=d_{0}+r_{0}$. $W_{1}$ is shown to be the sum of the contributions of the actual source located at $\mathbf{s}_{0}(0, h)$ with magnitude $P_{\text {inj }}$, and the image source at $\mathbf{s}_{1}(0,-h)$ with magnitude $R_{1}\left(\theta_{1}\right) P_{\text {inj }}$. Notice that the image source magnitude is not uniform, but accounts for the filtering effect of the reflection efficiency $R_{1}\left(\theta_{1}\right)$.

Now consider any point $\mathbf{r}(x, y)$ in plate 2 . According to equation (2), the energy density $W_{2}$ is

$$
W_{2}(\mathbf{r})=\int_{-\infty}^{\infty} \sigma_{2}\left(\nu, \theta_{2}\right) \frac{e^{-m_{2} d_{\nu}}}{2 \pi c_{2} d_{\nu}} d \nu
$$

with $d_{\nu}=|\mathbf{p}-\mathbf{r}|=\left[(x-\nu)^{2}+y^{2}\right]^{1 / 2} . \theta_{2}$ is the emission angle at $\mathbf{p}$ towards $\mathbf{r}$.

$$
W_{2}(\mathbf{r})=\frac{P_{\text {inj }}}{2 \pi c_{2}} \frac{c_{1}^{\prime}}{c_{2}^{\prime}} \int_{-\infty}^{\infty} T_{1}\left(\theta_{1}\right) \frac{e^{-m_{1} r_{\nu}}}{r_{\nu}} \delta_{\varphi_{\nu}}\left(\theta_{1}\right) \frac{e^{-m_{2} d_{\nu}}}{d_{\nu}} \cos \theta_{2} d \nu .
$$

$\theta_{1}$ is the angle related to $\theta_{2}$ with the Snell law of refraction. Now the condition $\psi_{\nu}=0$ states that the incident angle $\varphi_{\nu_{0}}$ and the emission angle $\theta_{2}$ satisfy the Snell condition. Furthermore,

$$
\frac{d \psi_{\nu}}{d \nu}=\frac{d \theta_{1}}{d \theta_{2}} \frac{d \theta_{2}}{d \nu}-\frac{d \varphi_{\nu}}{d \nu}=\frac{c_{1}^{\prime}}{c_{2}^{\prime}} \frac{\cos \theta_{2}}{\cos \theta_{1}} \frac{-1 / y}{1+\left(\frac{\nu-x}{y}\right)^{2}}+\frac{1 / h}{1+\left(\frac{\nu}{h}\right)^{2}}=\frac{c_{1}^{\prime}}{c_{2}^{\prime}} \frac{\cos \theta_{2}}{\cos \theta_{1}} \frac{|y|}{d_{\nu}^{2}}+\frac{h}{r_{\nu}^{2}}
$$


Using the condition $y \leqslant 0$, we obtain for $W_{2}$,

$$
W_{2}(\mathbf{r})=\frac{P_{\mathrm{inj}}}{2 \pi c_{2}} \frac{c_{1}^{\prime}}{c_{2}^{\prime}}\left[T_{1}\left(\theta_{1}\right) \frac{e^{-m_{1} r_{0}}}{r_{0}} \frac{e^{-m_{2} d_{0}}}{d_{0}} \frac{\cos \theta_{2}}{\frac{c_{1}^{\prime}}{c_{2}^{\prime}} \frac{\cos \theta_{2}}{\cos \theta_{1}} \frac{|y|}{d_{0}^{2}}+\frac{h}{r_{0}^{2}}}\right] .
$$

With $\cos \theta_{1}=h / r_{0}$ and $\cos \theta_{2}=|y| / d_{0}$, it yields

$$
W_{2}(\mathbf{r})=\frac{P_{\mathrm{inj}}}{2 \pi c_{2}}\left[T_{1}\left(\theta_{1}\right) e^{-\left(m_{1} r_{0}+m_{2} d_{0}\right)} \frac{c_{1}^{\prime}}{c_{1}^{\prime} r_{0} \frac{\cos \theta_{2}}{\cos \theta_{1}}+c_{2}^{\prime} d_{0} \frac{\cos \theta_{1}}{\cos \theta_{2}}}\right] .
$$

This relationship is not related with an image source solution except when $c_{1}^{\prime}=c_{2}^{\prime}$, that is the plates have identical propagation properties. In this case, $\theta_{1}=\theta_{2}$ and equation (27) reduces to

$$
W_{2}(\mathbf{r})=\frac{P_{\mathrm{inj}}}{2 \pi c_{1}}\left[T_{1}\left(\theta_{1}\right) \frac{e^{-m_{1} q_{1}}}{q_{1}}\right],
$$

with $q_{1}=d_{0}+r_{0}$.

The second step is to solve the problem for the couple of square plates shown in Figure 1. Now, we reduce the problem to the particular case where $c_{1}^{\prime}=c_{2}^{\prime}$ and thus, the image source technique is valid.

\subsection{Two finite plates with identical propagation properties}

Since both plates of Figure 1 have identical propagation properties, incidence and transmission angles at the junction are equal. In addition, $R_{1}=R_{2}$ and $T_{1}=T_{2}$ and the subscripts will be omitted in what follows.

For finite plates, an infinite number of image sources contributes to the energy. A recursive procedure is used to compute the position, magnitude and directivity of the image sources which account for the successive reflections and transmissions [21]. At step $n$, the algorithm computes positions and magnitudes of all image sources of order $n+1$. Notice that due to the rectangular geometry of plates, distinct sources of same order may produce identical sources of higher order. For instance, a source which reflects at a corner successively to the right and to the upper edge or the upper and the right edge, gives rise to the same image source as depicted in Figure 4.

The mathematical reason is that two symmetries with respect to right-angles axes commute. In this case, the new similar sources are to be taken into account just one time.

Two important properties of rectangular billiards greatly simplify the determination of image sources [22]. The first one is that during its travel, a ray impinges on the interface always with the same incidence $\theta$ as illustrated in Figure 5.

The magnitude of the corresponding image source is thus a product of terms $R(\theta)$ and $T(\theta)$ depending on the number of reflection and transmission through the interface. Other reflections do not modify the magnitude since the reflection efficiency of edges is assumed to be one. The second property of a rectangular billiard leads to splitting the problem into two separated steps. The first one is the determination of reflections along the $y$-axis and the second one is the determination of reflections and transmissions along the $x$-axis. Positions of image sources created by reflections along the $y$-axis are shown in Figure 6. 


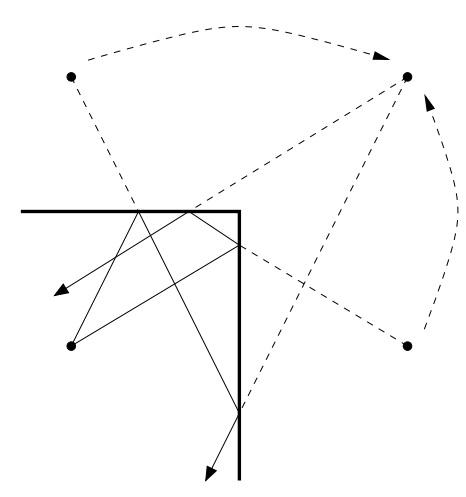

Figure 4: Identical image sources produced by two successive reflections on right-angled edges.

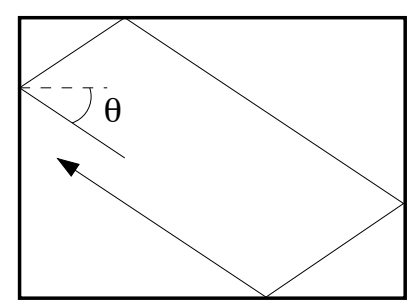

Figure 5: Reflections in a rectangular billiard.

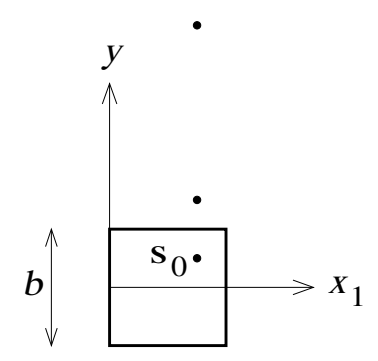

Figure 6: Image sources describing the reflections along the $y$-axis. 


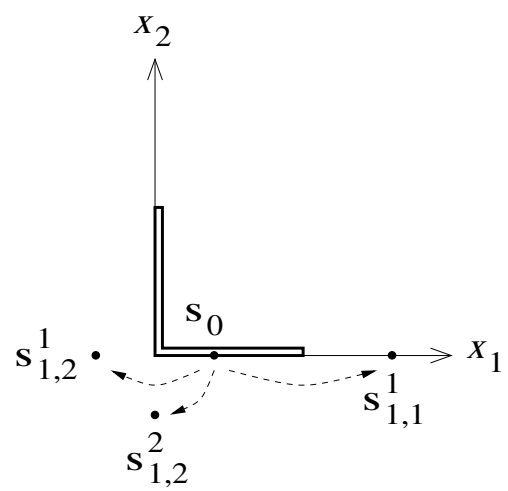

Figure 7: Image sources describing the first reflections and transmission of the actual source $\mathbf{s}_{0}$ along the $\left(x_{1}, x_{2}\right)$-axis.

They are easily computed by applying symmetries to the actual source with respect to the edges $y=b / 2$ and $y=-b / 2$ where $b$ is the width of plates. Since the reflection efficiency of edges is one, all these image sources have the same magnitude. Now, it may be considered that all image sources of Figure 6 reflect along the $x_{1}$-axis and $x_{2}$-axis as a group. The problem is thus confined into the $\left(x_{1}, x_{2}\right)$-plane.

From now on, we define the order of a source as the number reflections and transmissions along the $x_{1}, x_{2}$-axis without counting reflections along the $y$-axis. To start the algorithm, we consider that three events may occur to the actual source: a reflection on the right edge, a reflection on the interface and a transmission through the interface (Figure 7).

The first two event give rise to image sources located along the $x_{1}$-axis and obtained by applying symmetries with respect to the right edge and the interface. The magnitude of the first one is unchanged and the magnitude of the second one is $R(\theta)$. They are the only image sources of first order acting on the plate 1 and they are denoted $\mathbf{s}_{1, i}^{1}, i=1,2$ with magnitudes $A_{1,1}^{1}=1$ and $A_{1,2}^{1}=R(\theta)$. The upperscript denotes the plate of interest, the first subscript is the order of the source and the second subscript runs over 1,2. The third event is the transmission through the interface. The source now acts on the second plate. The corresponding image source is obtained by turning down the actual source on the $x_{2}$-axis. It is denoted $\mathbf{s}_{1,1}^{2}$ with a magnitude $A_{1,1}^{2}=T(\theta)$ (Figure 7).

Now, it is apparent that all image sources lying on the $x_{1}$-axis act on plate 1 whereas those sources located on $x_{2}$-axis only act on plate 2 . At step $n$ of the algorithm, several events (reflection and transmission) may occur to sources of order $n$. The nature of these events depends on the position of the sources. A source located on the right of the interface (Figure 8a) may be

- reflected by the interface and then submitted a symmetry with respect to the interface, its magnitude is multiplied by $R(\theta)$,

- transmitted through the interface and then is turned down to the $x_{2}$-axis, its magnitude is multiplied by $T(\theta)$.

A source located on the left of the interface (Figure 8b) may only be reflected by the right edge and then submitted to a symmetry with respect to that edge with an unchanged magnitude. A 


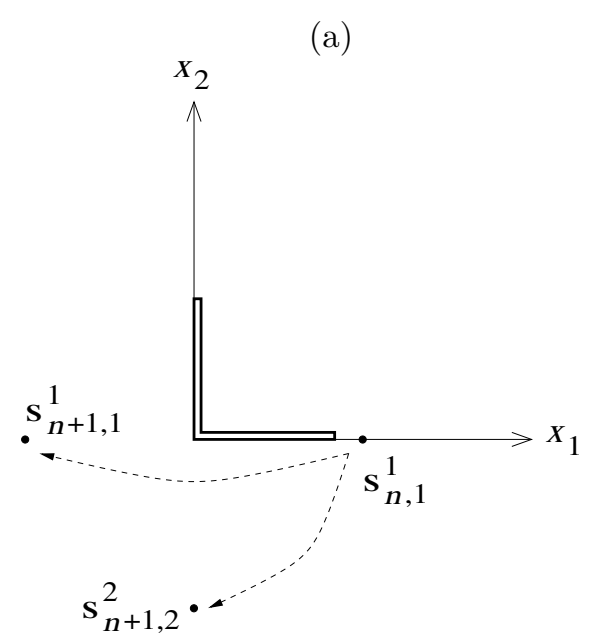

(c)

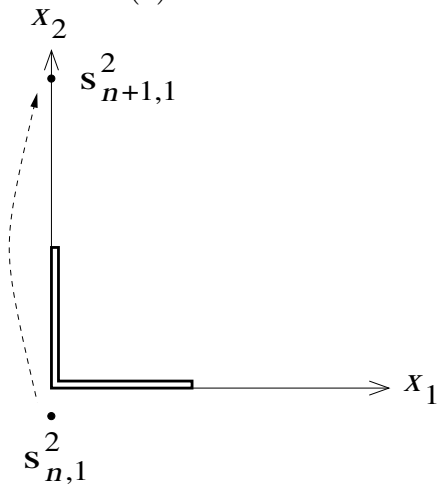

(b)

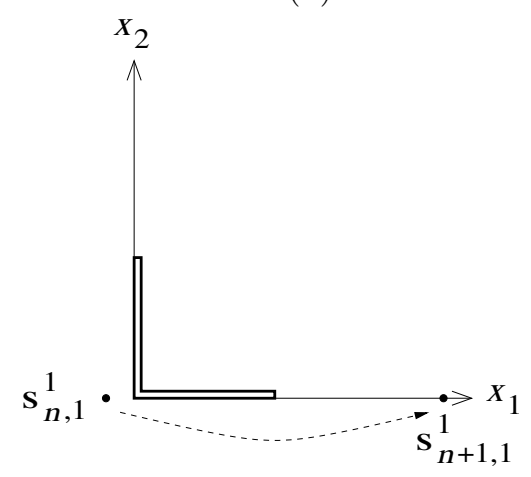

(d)

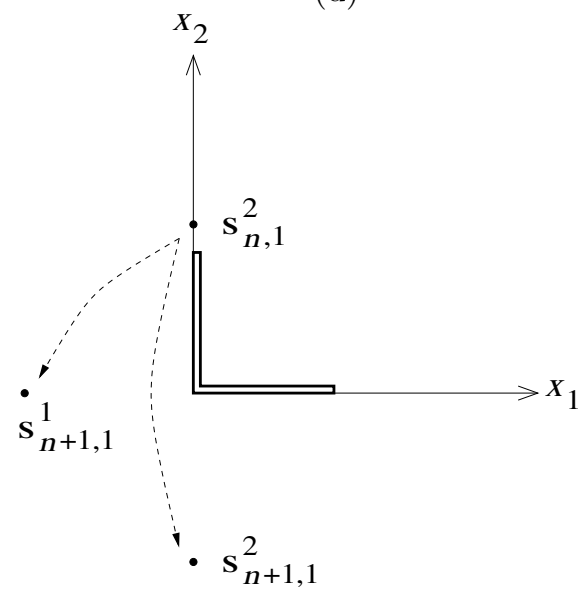

Figure 8: Image sources describing the reflections and transmission of the image source $\mathbf{s}_{n, 1}^{i}$ along the $\left(x_{1}, x_{2}\right)$-axis. 


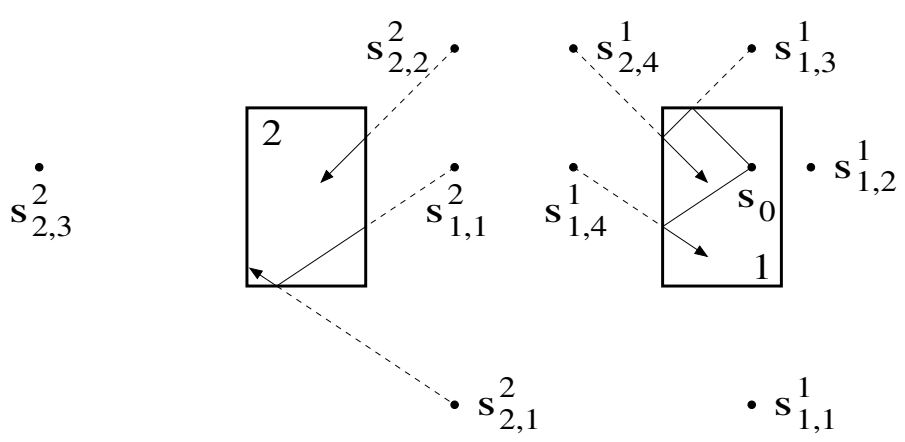

Figure 9: Image sources for the specular law of transmission and reflection between for two finite plates.

source located below the interface (Figure 8c) may only be reflected on the upper edge with an unchanged magnitude. Finally, a source located above the interface (Figure 8d) may be

- reflected by the interface, its magnitude is multiplied by $R(\theta)$,

- transmitted through the interface and then is turned down to the $x_{1}$-axis, with its magnitude multiplied by $T(\theta)$.

By recursively applying this algorithm, all the image sources that is, all the paths between the actual source and any point on plate 1 or 2 are determined.

We note $\mathbf{s}_{n, k}^{i}$ and $A_{n, k}^{i}(\theta)$ the position and magnitude of the $k^{t h}$ image source of order $n$ acting on plate $i$. $\theta$ denotes the angle between the vector $\mathbf{r}-\mathbf{s}_{n, k}^{1}$ from the source to the observation point and the outside normal to the interface $\mathbf{n}_{i}$. Now, $k$ runs over $-\infty$ to $\infty$ to take into account the effect of reflections along the $y$-axis. The sources of order 0 and 1 and some sources of order 2 are shown in Figure 9.

At any point $\mathbf{r}$ in plate 1 , the energy is the sum of all contributions of the actual source $\mathbf{s}_{0}$ and image sources $\mathbf{s}_{n, k}^{1}$,

$$
W_{1}(\mathbf{r})=P_{\mathrm{inj}} G_{1}\left(\mathbf{s}_{0}, \mathbf{r}\right)+\sum_{n=1}^{\infty} \sum_{k=-\infty}^{\infty} A_{n, k}^{1}(\theta) G_{1}\left(\mathbf{s}_{n, k}^{1}, \mathbf{r}\right) .
$$

Similarly, for plate 2,

$$
W_{2}(\mathbf{r})=\sum_{n=1}^{\infty} \sum_{k=-\infty}^{\infty} A_{n, k}^{2}(\theta) G_{2}\left(\mathbf{s}_{n, k}^{2}, \mathbf{r}\right) .
$$

In these expressions, the angle $\theta$ depends on the location of the source $\mathbf{s}_{n, k}^{i}$ and observation point r.

\section{$5 \quad$ Results and discussion}

Energy fields of the two right-angled plates of Figure 1 are now evaluated by both reference and energy approaches. The calculations are summarized in the next section. Then the global energy 
level and the energy distribution inside each plate are investigated.

\subsection{The calculated system}

Applications have been performed with two identical aluminum plates $\left(\rho=2800 \mathrm{~kg} \mathrm{~m}^{-3}, \nu=\right.$ $0.3, E=7210^{9} \mathrm{~N} \mathrm{~m}^{-2}$ ). The plates are $1 \mathrm{~m}$ square, of thickness $10^{-3} \mathrm{~m}$, clamped on the sides parallel to the junction and simply supported on the other sides. The plate 1 is excited by a transverse force of $1 N$ at the point $\mathbf{s}_{0}$ given by $x_{1}=0.2 \mathrm{~m}, y=0.3 \mathrm{~m}$. Both plates are damped with a loss factor $\eta=1 \%$. The calculation frequency is chosen in order to get 40 flexural wavelengths within the plates, thus $f=16000 \mathrm{~Hz}$.

The reference result is the numerical solution for the Love-Kirchhoff's plate equations with the relevant boundary and coupling conditions. It is derived using the approach developed in reference [23] with the same assumption that plates are rigid in-plane, i.e in-plane displacements are neglected. Due to the simply supported boundary conditions, the displacement field is thought of as the sum of sine functions in the $y$ direction. A wave decomposition is applied in the $x_{1,2}$ directions. Like SEA, integral energy approaches give an averaged estimation of the real response, and three reference calculations have consequently been performed depending on the kind of average that is achieved: since frequency, material properties and geometry can be considered as being inaccurately known, the energy has been averaged over the frequency, $\omega \in\left[\omega_{c}-\Delta \omega / 2, \omega_{c}+\Delta \omega / 2\right]$, the Young's modulus, $E \in\left[E_{c}-\Delta E / 2, E_{c}+\Delta E / 2\right]$, and the excitation location, $\left.\mathbf{s}_{0} \in\left[\mathbf{s}_{0 c}-\Delta \mathbf{s}_{0} / 2, \mathbf{s}_{0 c}+\Delta \mathbf{s}_{0} / 2\right]\right)$. The subscript $c$ denotes the center value of the variables given below. In each case, the range of variation is calculated to get a variation of one wavelength in the smallest distance between two sources. For the present system, this smallest distance is between the direct source on plate 1 and the boundary $x_{1}=0 \mathrm{~m}$.

Energy calculations use the injected power of equation (4), and the efficiencies at the junction evaluated with the wave decomposition of reference [17], by keeping in mind that plates are assumed to be rigid in-plane. Since both plates have identical propagation properties, $R_{1}=R_{2}$ and $T_{1}=T_{2}$ and the subscripts may be omitted. The variations of $R(\theta)$ and $T(\theta)$ at the frequency of excitation $(16000 \mathrm{~Hz})$ are shown in Figure 10, where it can be seen that the transmission is higher for low angles.

The integral energy formulation with the specular directivity is derived with the image source method described in section 4.2. The level $N$ of the image sources to be considered in order to get a good estimation of the energies is defined by considering that sources of level higher than $N$ have negligible contributions. The criteria given in reference [24] is expressed in terms of the percentage $P$ of energy which should have been attenuated, the average absorption coefficient at boundaries $\alpha$, the mean free path of the plates $l$, and the attenuation coefficient $m=\eta \omega / c$ : $N=\ln (1-P) /[\ln (1-\alpha)-m l]$. In the present application, no absorption is to be considered and the mean free path for plates is given in [17] by $l=\pi S / L$, where $S$ and $L$ denote the surface and perimeter of plate. By choosing $P=99.99 \%$, one obtains for the present calculation $N=10$. Note that due to the frequency dependence of the attenuation coefficient, the required CPU-time for the image source approach reduces as the frequency increases, which is a very interesting feature compared to reference calculations.

For the integral energy formulation with the Lambert directivity, the collocation algorithm of section 3 with 10 elements per side of plate was used. Equations $(8,9)$ are consequently derived by solving an $80^{*} 80$ linear system. It is not required for the size of the boundary elements to be 


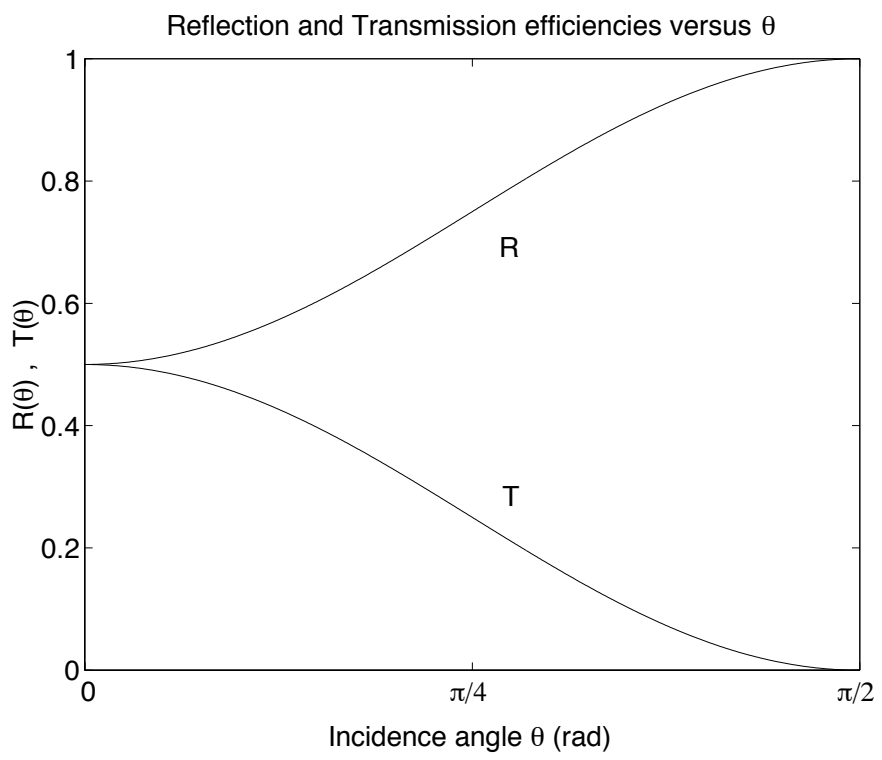

Figure 10: Evolution of the reflection and transmission efficiencies, $R(\theta)$ and $T(\theta)$ at the junction versus the incidence angle, at $16000 \mathrm{~Hz}$.

small compared to the flexural wavelength, since the describing variable is the averaged energy whom variations are expected to extend over much more than one wavelength. The size of the element is only related to the non-diffuse characteristic of the energy field which is directly related to the damping and geometry. As a result, while the required CPU-time for the reference calculation increases with the frequency, it remains quite stable for the integral energy approach. However, both integral energy approaches remain more time expensive than SEA and require a better knowledge of the system. Note that the low computation time is not the only interest of the energy approaches that provide a new intuitive tool to analyze the vibrations of structures at high frequencies.

Some SEA calculations have been performed using the same injected power (4) and efficiencies. By noting $E_{i}$ the total energy of the plate $i$, the global power balances for both plates are written

$$
\left\{\begin{array}{c}
P_{\mathrm{inj}}=\eta \omega E_{1}+\eta_{12} \omega E_{1}-\eta_{21} \omega E_{2}, \\
0=\eta \omega E_{2}+\eta_{21} \omega E_{2}-\eta_{12} \omega E_{1},
\end{array}\right.
$$

where $\eta_{12}$ and $\eta_{21}$ are the coupling loss factors. Since the plates are identical, $\eta_{12}$ and $\eta_{21}$ are equal. They are expressed in terms of the transmission efficiency by the integral over all incidence angles,

$$
\eta_{12}=\eta_{21}=\frac{L c}{\omega \pi S} \int_{0}^{\pi / 2} T(\theta) \cos \theta d \theta,
$$

where $L$ is the junction length, $c$ the group speed of flexural waves and $S$ the surface of the plates. 


\begin{tabular}{c|ccc|ccc}
\hline & Reference $\langle\omega\rangle$ & Reference $\langle E\rangle$ & Reference $\left\langle\mathbf{s}_{0}\right\rangle$ & SEA & Specular & Lambert \\
\hline$P_{\text {inj }}(m W)$ & 14.54 & 14.74 & 14.74 & 14.55 & 14.55 & 14.55 \\
\hline$E_{1}(d B)$ & 31.07 & 31.11 & 31.07 & 31.36 & 31.06 & 31.02 \\
\hline$E_{2}(d B)$ & 21.82 & 21.82 & 22.10 & 19.04 & 21.62 & 21.94 \\
\hline
\end{tabular}

Table 1: Injected power and total energy in both plates, by the three reference calculations, the SEA and the two integral energy approaches.

\subsection{Global energy}

The injected power and the total energy stored in each plate are shown in Table 1 for reference, energy and SEA results. Energies $E_{1}$ and $E_{2}$ are the integrals of the energy densities $W_{1}$ and $W_{2}$ given by the reference calculation and the integral energy approaches in equations $(29,30,10)$. They are expressed in $\mathrm{dB}\left(\right.$ re $\left.10^{-8} \mathrm{~J}\right)$.

It is shown that the averaged injected powers by the three reference calculations are in good agreement with that given by equation (4). Concerning the energies, both integral energy results are very closed to the reference ones. The SEA results are less accurate, which was expected since the plates are highly damped. In particular, the transmitted energy in plate 2 is underestimated because of the importance of the direct field contribution in plate 1: since the direct source is closed to the junction, the average energy on the plate 1 as predicted by the SEA is less than the real energy on the junction. Consequently, the predicted level of transmitted energy is lower. The spatial description of energy by the integral energy approaches succeeds in improving the prediction.

Note that the information on the strength of damping is rather given by the attenuation coefficient $m$ than by the damping coefficient $\eta$. Indeed, the attenuation coefficient is directly involved in the decreasing of energy of rays as shown by equations (1). In the present case, the attenuation produced by damping over the mean free path $l$ is $e^{-m l} \sim 0.36$, meaning that in average, one third of the energy is attenuated between two reflections of rays.

\subsection{Distribution of energy}

Contour plots of the flexural energy repartition in both plates by reference and integral energy calculations are shown in Figure 11. The plate 1, directly excited, is on the right. Energy densities are expressed in $\mathrm{dB}\left(\mathrm{re} 10^{-8} \mathrm{~J} \mathrm{~m}^{-2}\right)$. The reference calculation corresponds to the frequencyaveraged results. Anyway, the three averages lead to very similar results.

In both plates the energy field is shown to be not diffuse, with more than $6 \mathrm{~dB}$ of variation in each plate. These variations are well described by the energy flow approaches. One easily recognizes the cylindrical pattern of the direct field due to the excitation in plate 1 . About the energy field in plate 2, one may observe that the specular directivity leads to a slightly better description than the diffuse one. Due to the proximity of the direct source in plate 1, the upperright corner of plate $2\left(x_{2}=0 \mathrm{~m}, y=0.5 \mathrm{~m}\right)$ shows the higher energy level, which is predicted by both energy approaches. However, the energy density does not have a cylindrical shape centered on the upper-right corner as predicted by the diffuse directivity. The specular law provides a more reliable description. The reason is that the specular law accurately accounts for the filtering effect 

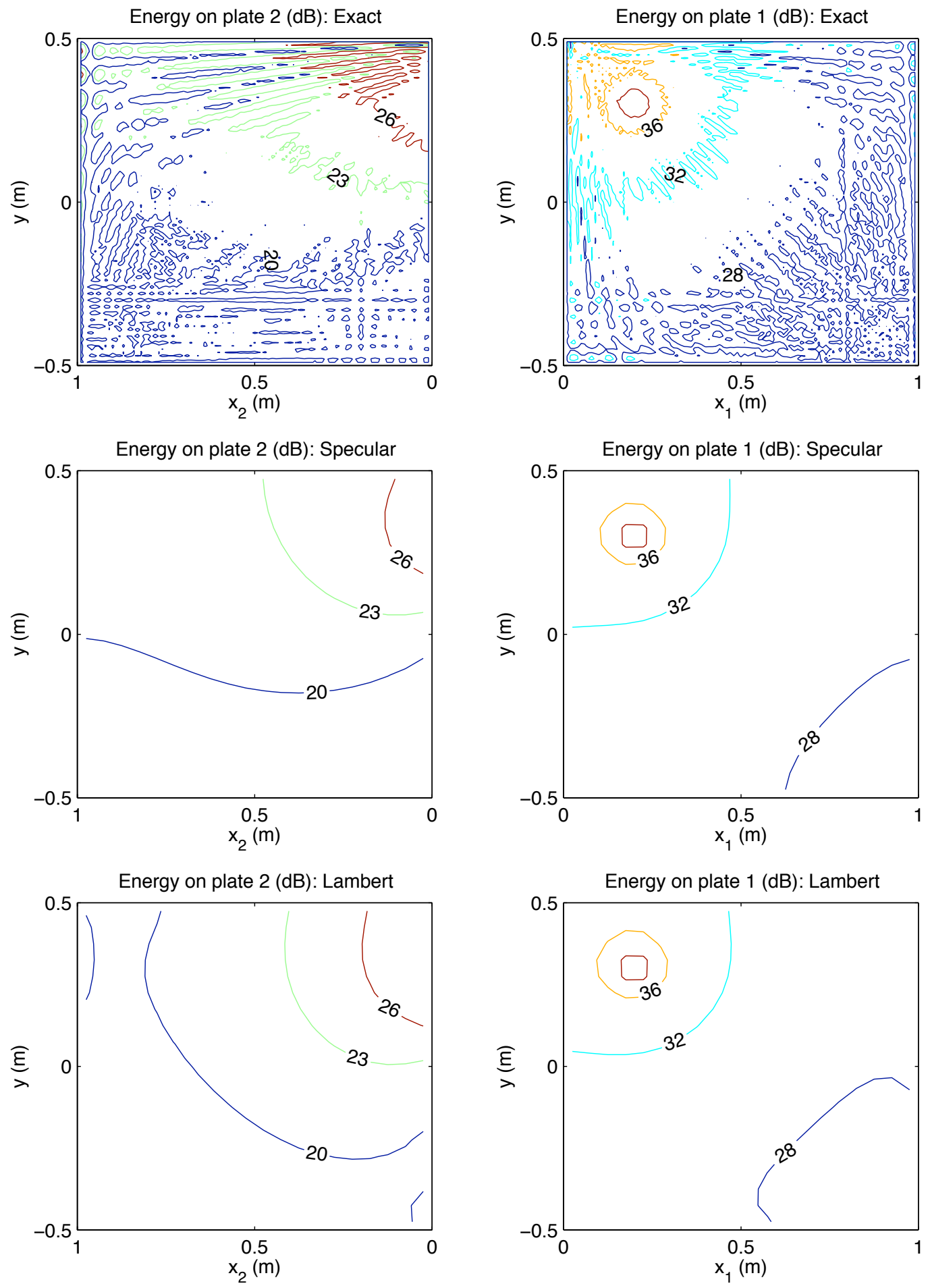

Figure 11: Flexural energy distribution in both plates, in $\mathrm{dB}\left(\right.$ re $10^{-8} \mathrm{~J} \mathrm{~m}^{-2}$ ). The reference frequency averaged result, and the energy flow results with specular and Lambert directivities are presented. The SEA gives $W_{1}=31.36 d B$ and $W_{2}=19.04 d B$. 
of the junction: the largest part of the transmitted energy in plate 2 comes from the part of plate 1 around the excitation where the energy level is the higher. Since the transmission efficiency is lower for large incidence angles (see Figure 10), the energy transmitted in plate 2 is lower on the low part of plate $2(y<0)$ than in the upper part $(y>0)$. This effect is well predicted by the integral energy approach with the specular directivity.

For both energy flow approaches, the prediction in the vicinity of the driving point and the boundaries is incorrect because near-field terms are neglected in the description. One may consider that a distance of one wavelength is enough for this terms to be negligible, which gives $0.025 \mathrm{~m}$ in the present case.

\section{Conclusion}

The presented integral energy flow approach is shown to be particularly suitable for damped systems at high frequency, when energy fields are not diffuse. The main assumptions of the method are the decomposition in uncorrelated cylindrical waves, and the kind of reflection-transmission law to be used at boundaries. It is shown that both specular and Lambert laws lead to an accurate estimation of the averaged energy fields. The specular law seems to give a better description of the distribution of energy since it accounts for the filtering effect due to the dependence of reflection and transmission efficiencies on the incidence angle.

Compared to the SEA, the integral energy approaches require more CPU-time as well as a more complete knowledge of the system. As a result, they lead to a more reliable description of the system and give the distribution of energy inside subsystems. Compared to an reference resolution, the integral energy approaches give averaged values of the energy density, with a great gain of CPU-time. They also provide an interesting tool to analyze the vibrations of structures at high frequencies.

Although the diffuse and specular versions of the energy flow approach only differ by the assumed directivity of reflected and transmitted rays at boundaries, they lead to completely distinct mathematical resolutions. It is shown in this paper how the image source method is suitable to solve the specular case. However, even if the image source method may be extended to non rectangular geometries as was done for acoustic rooms in [21], it is applicable only when the coupled plates have the same propagation properties. In other cases, ray or boundary element approaches may be used to solve the equations for the specular reflection $[12,15]$. On the other hand, the integral equation derived with the diffuse reflection is general and may be easily solved for any geometries and material of coupled plates by using the standard collocation procedure described in this paper.

\section{Acknowledgments}

This work was supported a Lavoisier fellowship of the Ministère des Affaires Étrangères Français (French Ministry of Foreign Affairs). 


\section{References}

[1] R.H. Lyon and R.G. DeJong. Theory and Applications of Statistical Energy Analysis. Butterworth-Heinemann, USA, 1995.

[2] D.J. Nefske and S.H. Sung. Power flow finite element analysis of dynamic systems: basic theory and application to beams. Transactions of ASME, Journal of Vibration, Acoustics, Stress, and Reliability in Design, 111:94-100, 1989.

[3] O.M. Bouthier and R.J. Bernhard. Simple models of the energetics of transversely vibrating plates. Journal of Sound and Vibration, 182(1):149-164 (doi: 10.1006/jsvi.1995.0187), 1995.

[4] R.S. LANGLEY. On the vibrational conductivity approach to high frequency dynamics for two-dimensional structural components. Journal of Sound and Vibration, 182(4):637-657 (doi: 10.1006/jsvi.1995.0223), 1995.

[5] A. Le Bot. Geometric diffusion of the energy and comparison with the vibrational conductivity approach. Journal of Sound and Vibration, 212(4):637-647 (doi: 10.1006/jsvi.1997.1442), 1998.

[6] H. Kuttruff. Energetic sound propagation in rooms. Acustica, 83(4):622-628, 1997.

[7] R.N. MiLES. Sound field in a rectangular enclosure with diffusely reflecting boundary. Journal of Sound and Vibration, 92(2):203-226, 1984.

[8] A. Le Bot. A vibroacoustic model for high frequency analysis. Journal of Sound and Vibration, 211(4):537-554 (doi: 10.1006/jsvi.1997.1378), 1998.

[9] A. Le Bot and A. Bocquillet. Comparison of an integral equation on energy and the raytracing technique in room acoustics. Journal of Acoustical Society of America, 108(4):17321740, 2000.

[10] V. Cotoni, A. Le Bot, and L. Jezequel. Vibroacoustique hautes fréquences : modèle énergétique local pour le rayonnement. Canadian Acoustic - Acoustique Canadienne (proceedings of Acoustics Week In Canada), 28(3), 2000.

[11] A. LE Bot. Energy transfer for high frequencies in built-up structures. Journal of Sound and Vibration, 250(2):247-275 (doi: 10.1006/jsvi.2001.3933), 2002.

[12] L.P. Franzoni, D.B. Bliss, and J.W. Rouse. An acoustic boundary element method based on energy and intensity variables for prediction of high-frequency broadband sound fields. Journal of Acoustical Society of America, 110(6):3071-3081, 2001.

[13] A. Le Bot. A functional equation for the specular reflection of rays. submitted to the Journal of Acoustical Society of America, 2001.

[14] V. Cotoni, A. Le Bot, and L. Jezequel. High-frequency radiation of L-shaped plate by a local energy flow approach. Journal of Sound and Vibration, 250(3):431-444 (doi: 10.1006/jsvi.2001.3952), 2002.

[15] K.S. ChAe and J.G. IH. Prediction of vibrational energy distribution in the thin plate at high-frequency bands by using the ray tracing method. Journal of Sound and Vibration, 240(2):263-292 (doi: 10.1006/jsvi.2000.3231), 2001. 
[16] J. KANG. Sound propagation in street canyons: Comparison between diffusely and geometrically reflecting boundaries. Journal of Acoustical Society of America, 107(3):1394-1404, 2000.

[17] L. Cremer, M. Heckl, and E.E. Ungar. Structure-Borne Sound. Springer-Verlag, 1985.

[18] D. Bouche, F. Molinet, and R. Mittra. Asymptotic methods in electromagnetics. Springer-Verlag, 1997.

[19] W. Wöhle, T. Beckmann, and H. Schreckenbach. Coupling loss factors for statistical energy analysis of sound transmission at rectangular slab joints, part. I. Journal of Sound and Vibration, 77(3):323-334, 1981.

[20] H. Kuttruff. Room acoustics. (2nd ed.) Applied Science Publishers, London, UK, 1979.

[21] J. Borish. Extension of the image model to arbitrary polyhedra. Journal of Acoustical Society of America, 75(6):1827-1836, 1984.

[22] M. Berger. La mathématique du billard. Pour la Science, 163:76-85, 1991.

[23] R.S. Langley. Application of the dynamic stiffness method to the free and forced vibrations of aircraft panels. Journal of Sound and Vibration, 135(2):319-331, 1989.

[24] S.M. DAnCE and B.M. Shield. The complete image-source method for the prediction of sound distribution in non-diffuse enclosed spaces. Journal of Sound and Vibration, 201(4):473489 (doi: 10.1006/jsvi.1996.0770), 1997. 